\title{
Algebraic sums and products of univoque bases
}

\author{
Karma Dajani ${ }^{\mathrm{a}}$, Vilmos Komornik ${ }^{\mathrm{b}}$, Derong Kong ${ }^{\mathrm{c}, *}$, Wenxia $\mathrm{Li}^{\mathrm{d}}$ \\ ${ }^{a}$ Department of Mathematics, Utrecht University, Fac Wiskunde en informatica and MRI, Budapestlaan \\ 6, P.O. Box 80.000, 3508 TA Utrecht, The Netherlands \\ ${ }^{b}$ Département de mathématique, Université de Strasbourg, 7 rue René Descartes, 67084 Strasbourg \\ Cedex, France \\ ${ }^{c}$ Mathematical Institute, University of Leiden, PO Box 9512, 2300 RA Leiden, The Netherlands \\ ${ }^{d}$ Department of Mathematics, Shanghai Key Laboratory of PMMP, East China Normal University, \\ Shanghai 200062, People's Republic of China
}

\begin{abstract}
Given $x \in(0,1]$, let $\mathcal{U}(x)$ be the set of bases $q \in(1,2]$ for which there exists a unique sequence $\left(d_{i}\right)$ of zeros and ones such that $x=\sum_{i=1}^{\infty} d_{i} / q^{i}$. Lü, Tan and Wu [15] proved that $\mathcal{U}(x)$ is a Lebesgue null set of full Hausdorff dimension. In this paper, we show that the algebraic sum $\mathcal{U}(x)+\lambda \mathcal{U}(x)$ and product $\mathcal{U}(x) \cdot \mathcal{U}(x)^{\lambda}$ contain an interval for all $x \in(0,1]$ and $\lambda \neq 0$. As an application we show that the same phenomenon occurs for the set of non-matching parameters studied by the first author and Kalle [2].

Keywords: Algebraic differences, non-integer base expansions, univoque bases, thickness, Cantor sets, non-matching parameters.

2010 MSC: 28A80, 11A63, 37B10
\end{abstract}

\section{Introduction}

Non-integer base expansions, a natural extension of dyadic expansions, have got much attention since the ground-breaking works of Rényi [18] and Parry [17]. Given a base $q \in(1,2]$, an infinite sequence $\left(d_{i}\right)$ of zeros and ones is called a $q$-expansion of $x$ if

$$
x=\sum_{i=1}^{\infty} \frac{d_{i}}{q^{i}}=:\left(\left(d_{i}\right)\right)_{q} .
$$

A number $x$ has a $q$-expansion if and only if $x \in I_{q}:=\left[0, \frac{1}{q-1}\right]$. Contrary to the the dyadic expansions, Lebesgue almost every $x \in I_{q}$ has a continuum of $q$-expansions (see [19]). On the other hand, for each $k \in \mathbb{N}:=\{1,2, \ldots\}$ or $k=\aleph_{0}$ there exist $q \in(1,2]$ and $x \in I_{q}$ such that $x$ has precisely $k$ different $q$-expansions (see [6]). For more information on the non-integer base expansions we refer to the survey paper [7] and the book chapter [4].

\footnotetext{
${ }^{*}$ Corresponding author

Email addresses: k.dajani1@uu.nl (Karma Dajani), komornik@math.unistra.fr (Vilmos Komornik), derongkong@126.com (Derong Kong), wxli@math.ecnu.edu.cn (Wenxia Li)
} 
On the other hand, algebraic differences of Cantor sets and their connections with dynamical systems have been intensively investigated since the work of Newhouse [16], who introduced the notion of thickness to study whether a given Cantor set $C \subset \mathbb{R}$ has a nonempty intersection with its translations. Since $C \cap(C+t) \neq \emptyset$ if and only if $t \in C-C$, where the algebraic difference of two sets $A, B \subset \mathbb{R}$ is defined by $A-B:=\{a-b: a \in A, b \in B\}$, the thickness (see Definition 3.1 below) can be used to study the algebraic difference of Cantor sets (cf. [1, 13, 14]).

In this paper, we consider the algebraic differences of sets of univoque bases for given real numbers. To be more precise, for $x \in(0,1]$, let $\mathcal{U}(x)$ be the set of bases $q \in(1,2]$ such that $x$ has a unique $q$-expansion. Then each element of $\mathcal{U}(x)$ is called a univoque base of $x$. Lü et al. [15] proved that $\mathcal{U}(x)$ is a Lebesgue null set of full Hausdorff dimension.

We will prove the following result for the algebraic sum and product of $\mathcal{U}(x)$ defined respectively by

$$
\mathcal{U}(x)+\lambda \mathcal{U}(x):=\{p+\lambda q: p, q \in \mathcal{U}(x)\} \quad \text { and } \quad \mathcal{U}(x) \cdot \mathcal{U}(x)^{\lambda}:=\left\{p q^{\lambda}: p, q \in \mathcal{U}(x)\right\} .
$$

Theorem 1.1. For every $x \in(0,1]$ and every $\lambda \neq 0$ both the sum $\mathcal{U}(x)+\lambda \mathcal{U}(x)$ and product $\mathcal{U}(x) \cdot \mathcal{U}(x)^{\lambda}$ contain an interval.

We mention that the product $\mathcal{U}(x) \cdot \mathcal{U}(x)^{\lambda}$ in Theorem 1.1 can be converted to a sum by taking the logarithm and then repeating the construction (see Section 3 for more details). Hence, we will focus more on the algebraic sum $\mathcal{U}(x)+\lambda \mathcal{U}(x)$.

Remarks 1.2 .

- For $\lambda=-1$ Theorem 1.1 states that the algebraic difference $\mathcal{U}(x)-\mathcal{U}(x)$ and quotient $\mathcal{U}(x) \cdot \mathcal{U}(x)^{-1}$ contain an interval for each $x \in(0,1]$.

- For $x=1$ the set $\mathcal{U}:=\mathcal{U}(1)$ is well-studied. For example, it has a smallest element $q_{K L} \approx 1.78723$, called the Komornik-Loreti constant (see [8]), and its closure $\overline{\mathcal{U}}$ is a Cantor set (see [9]). Furthermore, the local Hausdorff dimension of $\mathcal{U}$ is positive (see [12]), i.e., $\operatorname{dim}_{H}(\mathcal{U} \cap(q-\delta, q+\delta))>0$ for any $q \in \mathcal{U}$ and $\delta>0$. Theorem 1.1 for $x=1$ and $\lambda=-1$ states that the algebraic difference $\mathcal{U}-\mathcal{U}$ and quotient $\mathcal{U} \cdot \mathcal{U}^{-1}$ contain an interval.

- The algebraic sum $\mathcal{U}(x)+\lambda \mathcal{U}(x)$ containing an interval for all $\lambda \neq 0$ can also be expressed by saying that for each $x \in(0,1]$ and for each oblique straight line $L$ passing through 0 , the projection of the product set $\mathcal{U}(x) \times \mathcal{U}(x)=\{(p, q): p, q \in \mathcal{U}(x)\}$ onto $L$ contains an interval for all $x \in(0,1]$.

We will also show that the same phenomenon occurs for the set of non-matching parameters, recently studied by the first author and Kalle [2]. Let us introduce for each $\alpha \in[1,2]$ the map $S_{\alpha}:[-1,1] \rightarrow[-1,1]$ by the formula

$$
S_{\alpha}(x)=\left\{\begin{array}{lll}
2 x+\alpha, & \text { if } & -1 \leq x<\frac{1}{2} \\
2 x, & \text { if } & -\frac{1}{2} \leq x \leq \frac{1}{2} \\
2 x-\alpha, & \text { if } & \frac{1}{2}<x \leq 1
\end{array}\right.
$$


The parameter $\alpha$ is called a matching parameter if there exists $m \in \mathbb{N}$ such that $S_{\alpha}^{m}(1)=$ $S_{\alpha}^{m}(1-\alpha)$, and a non-matching parameter otherwise.

If $\alpha$ is a matching parameter, then the density $h_{\alpha}$ of the invariant measure with respect to $S_{\alpha}$ is simply a finite sum of indicator functions.

It was shown in [2] that the set $\mathcal{N}$ of all non-matching parameters is a Lebesgue null set of full Hausdorff dimension. We prove the following result:

Theorem 1.3. For every $\lambda \neq 0$ both the algebraic sum $\mathcal{N}+\lambda \mathcal{N}$ and product $\mathcal{N} \cdot \mathcal{N}^{\lambda}$ contain an interval.

The paper is organized as follows. In Section 2 we investigate the topological structure of $\mathcal{U}(x)$ and we construct a Cantor subset of $\mathcal{U}(x)$ in a symbolic way. In Section 3 , we prove Theorem 1.1 by using a theorem of Newhouse on the thickness, and its recent improvements by Astels [1] (see Lemmas 3.2 and 3.6 below). Section 4 is devoted to the proof of Theorem [1.3. In the final section we prove that neither the algebraic sum $\mathcal{U}(1)+\mathcal{U}(1)$, nor the product $\mathcal{U}(1) \cdot \mathcal{U}(1)$ is an interval, and we conjecture that both the algebraic difference $\mathcal{U}(1)-\mathcal{U}(1)$ and quotient $\mathcal{U}(1) \cdot \mathcal{U}(1)^{-1}$ are intervals.

\section{Topological structure of $\mathcal{U}(x)$}

Given $x \in(0,1]$, let $\Phi_{x}$ be the coding map defined by

$$
\Phi_{x}:(1,2] \rightarrow\{0,1\}^{\mathbb{N}} ; \quad q \mapsto\left(a_{i}\right),
$$

where $\left(a_{i}\right)$ is the quasi-greedy $q$-expansion of $x$, i.e., the lexicographically largest $q$-expansion of $x$ not ending with $0^{\infty}$. In this paper, we will use lexicographical order $\prec, \preccurlyeq, \succ$ and $\succcurlyeq$ between sequences in $\{0,1\}^{\mathbb{N}}$ defined in the natural way. The definitions imply that $\Phi_{x}$ is strictly increasing with respect to this lexicographical order. Therefore, we may define intervals in terms of their codings via $\Phi_{x}$. For example, the symbolic interval $\left[\left(a_{i}\right),\left(b_{i}\right)\right]$ with $\left(a_{i}\right),\left(b_{i}\right) \in\{0,1\}^{\mathbb{N}}$ corresponds to the closed interval $[p, q] \subset(1,2]$, where $p=\Phi_{x}^{-1}\left(\left(a_{i}\right)\right)$ and $q=\Phi_{x}^{-1}\left(\left(b_{i}\right)\right)$. We emphasize that not every sequence in $\left[\left(a_{i}\right),\left(b_{i}\right)\right]$ corresponds to a base in $[p, q]$. In other words, $\Phi_{x}([p, q])$ is a proper subset of $\left[\left(a_{i}\right),\left(b_{i}\right)\right]$.

Set

$$
\mathbf{U}(x):=\left\{\Phi_{x}(q): q \in \mathcal{U}(x)\right\} .
$$

Then $\Phi_{x}$ is a bijection between $\mathcal{U}(x)$ and $\mathbf{U}(x)$. So, instead of looking at the set $\mathcal{U}(x)$ of univoque bases we focus on the symbolic set $\mathbf{U}(x)$ of univoque sequences. In [15], Lü et al. proved that $\mathcal{U}(x)$ has more weight at the right endpoint $q=2$, i.e., $\lim _{\delta \rightarrow 0} \operatorname{dim}_{H}(\mathcal{U}(x) \cap$ $[2-\delta, 2])=1$, and for $q \in(1,2)$ we have $\lim _{\delta \rightarrow 0} \operatorname{dim}_{H}(\mathcal{U}(x) \cap[q-\delta, q+\delta])<1$. Accordingly, in the symbolic space the cylinder set

$$
C_{n}(x)=\left\{\left(a_{i}\right) \in \mathbf{U}(x): a_{1} \cdots a_{n}=x_{1} \cdots x_{n}\right\}
$$

has the same topological entropy as the whole set $\mathbf{U}(x)$ for any $n \geq 1$, where $\left(x_{i}\right)=\Phi_{x}(2)$ is the quasi-greedy dyadic expansion of $x$. Here for a set $X \subseteq\{0,1\}^{\mathbb{N}}$ its topological entropy 
$h(X)$ is defined by

$$
h(X):=\liminf _{k \rightarrow \infty} \frac{\log \left|B_{n}(X)\right|}{k},
$$

where $\left|B_{n}(X)\right|$ denotes the total number of length $n$ blocks appearing in sequences of $X$.

Motivated by this observation, we will construct a symbolic Cantor subset $\mathbf{U}_{n}(x)$ contained in the cylinder set $C_{n}(x)$ for all large integers $n$. In the next section we will show that the corresponding Cantor set $\mathcal{U}_{n}(x)=\Phi_{x}^{-1}\left(\mathbf{U}_{n}(x)\right)$ has a thickness larger than one for all large integers $n$, and implying that $\mathcal{U}_{n}(x)+\lambda \mathcal{U}_{n}(x)$ contains an interval for each $\lambda \neq 0$. Since $\mathcal{U}_{n}(x) \subset \mathcal{U}(x)$, this will prove Theorem 1.1.

The following result was implicitly given by Lü et al. [15, Section 4], and we refer to this article for more details.

Lemma 2.1. Fix $x \in(0,1]$ arbitrarily and set $\left(x_{i}\right):=\Phi_{x}(2)$. There exist $M \in \mathbb{N} \cup\{0\}$ and a strictly increasing sequence $\left(N_{j}\right) \subset\{3,4, \ldots\}$ such that the following conditions are satisfied for each $N_{j}$ :

(i) we have

$$
x_{M+N_{j}}=1 \text { and } \mathbf{U}_{N_{j}}(x) \subseteq \mathbf{U}(x),
$$

where $\mathbf{U}_{N_{j}}(x)$ is the set of sequences

$$
x_{1} \cdots x_{M+N_{j}} \varepsilon_{1} \varepsilon_{2} \cdots
$$

satisfying

$$
\varepsilon_{1}=0, \quad \text { and } \quad \varepsilon_{n+1} \cdots \varepsilon_{n+N_{j}} \notin\left\{0^{N_{j}}, 1^{N_{j}}\right\} \quad \text { for all } n \geq 0 ;
$$

(ii) we have $\left(c_{i}\right) \succcurlyeq 0^{M} 10^{\infty}$ for all sequences $\left(c_{i}\right) \in \mathbf{U}_{N_{j}}(x)$.

(iii) we have $\left(\left(1^{N_{j}-1} 0\right)^{\infty}\right)_{q} \leq 1$ for all bases $q \in \Phi_{x}^{-1}\left(\mathbf{U}_{N_{j}}(x)\right)$.

Before proving the lemma we mention that although the sets $\mathbf{U}_{N_{j}}(x)$ also depend on $M$, we omit this in the notation for simplicity, because in the rest of the paper $x$ and hence $M$ will be fixed.

Proof. Note that $\left(x_{i}\right)=\Phi_{x}(2)$ is the dyadic expansion of $x$ not ending with $0^{\infty}$. We distingush four cases.

(a) If $\left(x_{i}\right)=x_{1} \cdots x_{m} 01^{\infty}$ for some $m \geq 0$, then by [15] we have

$$
x_{1} \cdots x_{m} 01^{j+2} \varepsilon_{1} \varepsilon_{2} \cdots \in \mathbf{U}(x)
$$

for all $j \geq 1$, where $\varepsilon_{1}=0$, and for $N_{j}:=j+2 \geq 3$ we have $\varepsilon_{n+1} \cdots \varepsilon_{n+N_{j}} \notin\left\{0^{N_{j}}, 1^{N_{j}}\right\}$ for all $n \geq 0$. This yields (i) and (ii) by taking $M=m+1$. Furthermore, for each $q \in \Phi_{x}^{-1}\left(\mathbf{U}_{N_{j}}(x)\right)$ the inequality

$$
\sum_{i=1}^{N_{j}} \frac{1}{q^{i}}<1
$$


holds, and hence (iii) follows:

$$
\left(\left(1^{N_{j}-1} 0\right)^{\infty}\right)_{q}=\left(\sum_{i=1}^{N_{j}-1} \frac{1}{q^{i}}\right)\left(\sum_{i=0}^{\infty} \frac{1}{q^{i N_{j}}}\right)<\left(1-\frac{1}{q^{N_{j}}}\right)\left(\sum_{i=0}^{\infty} \frac{1}{q^{i N_{j}}}\right)=1 .
$$

(b) If $\left(x_{i}\right)=1^{\infty}$, then $x=1$. By a similar argument as in (a) it follows that

$$
1^{j+2} \varepsilon_{1} \varepsilon_{2} \cdots \in \mathbf{U}(x)
$$

for any $j \geq 1$, where $\varepsilon_{1}=0$, and for $N_{j}:=j+2 \geq 3$ we have $\varepsilon_{n+1} \cdots \varepsilon_{n+N_{j}} \notin\left\{0^{N_{j}}, 1^{N_{j}}\right\}$ for all $n \geq 0$. This proves (i) and (ii) by taking $M=0$. Furthermore, for any $q \in \Phi_{x}^{-1}\left(\mathbf{U}_{N_{j}}(x)\right)$ we have

$$
\sum_{i=1}^{N_{j}} \frac{1}{q^{i}}<x=1
$$

this yields (iii) as above.

(c) If $\left(x_{i}\right)=1^{r_{1}} 0^{s_{1}} 1^{r_{2}} 0^{s_{2}} \cdots 1^{r_{k}} 0^{s_{k}} \ldots$ with $r_{k}, s_{k} \geq 1$ for all $k \geq 1$, then by [15] we deduce that

$$
1^{r_{1}} 0^{s_{1}} \cdots 1^{r_{j+2}} 0^{s_{j+2}} 01 \varepsilon_{1} \varepsilon_{2} \cdots \in \mathbf{U}(x)
$$

for all $j \geq 1$, where $\varepsilon_{1}=0$ and for $N_{j}:=r_{1}+s_{1}+\cdots+r_{j+2}+s_{j+2}-2 \geq 4$ we have $\varepsilon_{n+1} \cdots \varepsilon_{n+N_{j}} \notin\left\{0^{N_{j}}, 1^{N_{j}}\right\}$ for all $n \geq 0$. Therefore, (i) and (ii) follow by taking $M=4$. Furthermore, (iii) holds as in the preceding cases because

$$
\sum_{i=1}^{N_{j}} \frac{1}{q^{i}}<1
$$

for all $q \in \Phi_{x}^{-1}\left(\mathbf{U}_{N_{j}}(x)\right)$.

(d) If $\left(x_{i}\right)=0^{r_{1}} 1^{s_{1}} 0^{r_{2}} 1^{s_{2}} \cdots 0^{r_{k}} 1^{s_{k}} \ldots$ with $r_{k}, s_{k} \geq 1$ for all $k \geq 1$, then by [15] we have

$$
0^{r_{1}} 1^{s_{1}} \cdots 0^{r_{j+1}} 1^{s_{j+1}} 0^{r_{j+2}} 01 \varepsilon_{1} \varepsilon_{2} \cdots \in \mathbf{U}(x)
$$

for all $j \geq 1$, where $\varepsilon_{1}=0$, and for $N_{j}:=s_{1}+r_{2}+s_{2}+\cdots+r_{j+1}+s_{j+1}+r_{j+2}-1 \geq 3$ we have $\varepsilon_{n+1} \cdots \varepsilon_{n+N_{j}} \notin\left\{0^{N_{j}}, 1^{N_{j}}\right\}$ for all $n \geq 0$. This yields (i) and (ii) by taking $M=r_{1}+3$. Finally, (iii) holds again because

$$
\sum_{i=1}^{N_{j}} \frac{1}{q^{i}}<1
$$

for all $q \in \Phi_{x}^{-1}\left(\mathbf{U}_{N_{j}}(x)\right)$. 
Remark 2.2. Lemma 2.1 does not hold for $x>1$. Indeed, Lemma 2.1 (i) states that the set $\mathbf{U}(x)$ contains sequences with arbitrarily long blocks of consecutive zeros, and for this $\mathbf{U}(x)$ must contain bases arbitrarily close to 2: this follows from the usual lexicographic characterization of unique expansions. However, for $x>1$ the largest base for which $x$ has an expansion is $q_{x}:=1+1 / x<2$.

By Lemma 2.1 the tails of the sequences in $\mathbf{U}_{N_{j}}(x)$ contain neither $N_{j}$ consecutive zeros, nor $N_{j}$ consecutive ones. Furthermore, $\mathbf{U}_{N_{j}}(x) \subseteq \mathbf{U}(x)$ for all $x \in(0,1]$ and $j \geq 1$. Setting

we have

$$
\mathcal{U}_{N_{j}}(x):=\Phi_{x}^{-1}\left(\mathbf{U}_{N_{j}}(x)\right)=\left\{q \in(1,2]: \Phi_{x}(q) \in \mathbf{U}_{N_{j}}(x)\right\}
$$

$$
\mathcal{U}_{N_{j}}(x) \subseteq \mathcal{U}(x)
$$

for all $x \in(0,1]$ and $j \geq 1$. Hence the algebraic sum $\mathcal{U}(x)+\lambda \mathcal{U}(x)$ containing an interval will follow if we prove that the algebraic sum $\mathcal{U}_{N_{j}}(x)+\lambda \mathcal{U}_{N_{j}}(x)$ contains an interval for any fixed $\lambda \neq 0$, if $j \geq 1$ is sufficiently large. For this we will apply the results of Newhouse [16] and Astels [1]. Notice that $\mathcal{U}_{N_{j}}(x)$ is a Cantor set for any $x \in(0,1]$ and $j \geq 1$. In order to estimate the thickness of $\mathcal{U}_{N_{j}}(x)$ we need to describe its geometrical structure. For this we need to find an efficient way to construct $\mathcal{U}_{N_{j}}(x)$ by successively removing a sequence of open intervals from a closed interval.

Fix $x \in(0,1]$ and $j \geq 1$ arbitrarily. Since the coding map $\Phi_{x}$ defined in (2.1) is strictly increasing, each $q \in \mathcal{U}_{N_{j}}(x)$ may be encoded by a unique sequence $\Phi_{x}(q)=\left(a_{i}\right) \in \mathbf{U}_{N_{j}}(x)$. Conversely, each sequence $\left(a_{i}\right) \in \mathbf{U}_{N_{j}}(x)$ can be decoded to a unique base $q \in \mathcal{U}_{N_{j}}(x)$. Let $\left(x_{i}\right)=\Phi_{x}(2)$ be the dyadic expansion of $x$ not ending with $0^{\infty}$. Suppose that the integer $M$ and the sequence $\left(N_{j}\right)$ depending on $x$ are defined as in Lemma 2.1. Given $j \geq 1$, let $\Omega_{j}(x)$ be the set of all finite initial words of length larger than $M+N_{j}$ occurring in $\mathbf{U}_{N_{j}}(x)$, i.e.,

$$
\Omega_{j}(x)=\left\{\omega_{1} \cdots \omega_{n}: n>M+N_{j} \quad \text { and } \omega_{1} \cdots \omega_{n} c_{1} c_{2} \cdots \in \mathbf{U}_{N_{j}}(x) \text { for some }\left(c_{i}\right)\right\} .
$$

Since the tails of the sequences in $\mathbf{U}_{N_{j}}(x)$ contain neither $N_{j}$ consecutive zeros, nor $N_{j}$ consecutive ones, the words of $\Omega_{j}(x)$ are divided into $2 N_{j}-2$ disjoint classes: the words ending with $10^{k}$ and those ending with $01^{k}$ for some $k \in\left\{1,2, \ldots, N_{j}-1\right\}$.

Recall that a symbolic interval $\left[\left(a_{i}\right),\left(b_{i}\right)\right]$ corresponds to the closed interval $[p, q]$, if $\left(a_{i}\right)=\Phi_{x}(p)$ and $\left(b_{i}\right)=\Phi_{x}(q)$. For each $\omega \in \Omega_{j}(x)$ we denote by $\mathbf{I}_{\omega}$ the smallest symbolic interval containing all sequences of $\mathbf{U}_{N_{j}}(x)$ that begin with $\omega$. The following explicit description of these intervals follows directly from the definition of $\mathbf{U}_{N_{j}}(x)$.

Lemma 2.3. Let $\omega \in \Omega_{j}(x)$.

(i) If $\omega$ ends with $10^{k}$ for some $k \in\left\{1, \ldots, N_{j}-1\right\}$, then

$$
\mathbf{I}_{\omega}=\left[\omega 0^{N_{j}-1-k}\left(10^{N_{j}-1}\right)^{\infty}, \omega\left(1^{N_{j}-1} 0\right)^{\infty}\right] .
$$

(ii) If $\omega$ ends with $01^{k}$ for some $k \in\left\{1, \ldots, N_{j}-1\right\}$, then

$$
\mathbf{I}_{\omega}=\left[\omega\left(0^{N_{j}-1} 1\right)^{\infty}, \omega 1^{N_{j}-1-k}\left(01^{N_{j}-1}\right)^{\infty}\right] .
$$


By Lemma 2.1(i) all sequences in $\mathbf{U}_{N_{j}}(x)$ begin with $x_{1} \cdots x_{M+N_{j}} 0=x_{1} \cdots x_{M+N_{j}-1} 10$. Applying Lemma2.3(i) it follows that the smallest symbolic interval which contains $\mathbf{U}_{N_{j}}(x)$ is

$$
\mathbf{I}_{x_{1} \cdots x_{M+N_{j}} 0}=\left[x_{1} \cdots x_{M+N_{j}}\left(0^{N_{j}-1} 1\right)^{\infty}, x_{1} \cdots x_{M+N_{j}}\left(01^{N_{j}-1}\right)^{\infty}\right] .
$$

An immediate consequence of Lemma 2.3 is the following:

Lemma 2.4. Let $\omega \in \Omega_{j}(x)$.

(i) If $\omega$ ends with $10^{N_{j}-1}$, then

$$
\omega 0 \notin \Omega_{j}(x) \text { and } \quad \mathbf{I}_{\omega 1}=\mathbf{I}_{\omega} .
$$

(ii) If $\omega$ ends with $01^{N_{j}-1}$, then

$$
\omega 1 \notin \Omega_{j}(x) \quad \text { and } \quad \mathbf{I}_{\omega 0}=\mathbf{I}_{\omega} .
$$

(iii) In the remaining cases, $\mathbf{I}_{\omega}$ is the disjoint union of the non-empty intervals

$$
\mathbf{I}_{\omega 0}, \quad \mathbf{I}_{\omega 1} \text { and } \quad \mathbf{G}_{\omega}:=\mathbf{I}_{\omega} \backslash\left(\mathbf{I}_{\omega 0} \cup \mathbf{I}_{\omega 1}\right) .
$$

Now we may describe the geometrical structure of $\mathcal{U}_{N_{j}}(x)$. Given a symbolic interval $\mathbf{I}=\left[\left(a_{i}\right),\left(b_{i}\right)\right]$ with $\left(a_{i}\right),\left(b_{i}\right) \in \mathbf{U}_{N_{j}}(x)$, we denote by $I=[p, q]$ the corresponding interval in $\mathbb{R}$, where $p=\Phi_{x}^{-1}\left(\left(a_{i}\right)\right)$ and $q=\Phi_{x}^{-1}\left(\left(b_{i}\right)\right)$. Then the symbolic intervals $\mathbf{I}_{\omega}, \mathbf{G}_{\omega}$ are transferred to the real intervals $I_{\omega}, G_{\omega}$, respectively. Set

$$
\Omega_{j}^{*}(x):=\left\{\omega \in \Omega_{j}(x): G_{\omega} \neq \emptyset\right\} .
$$

Lemma 2.5. The non-empty open intervals $G_{\omega}, \omega \in \Omega_{j}^{*}(x)$ are pairwise disjoint, and

$$
\mathcal{U}_{N_{j}}(x)=I_{x_{1} \cdots x_{M+N_{j}} 0} \backslash \bigcup_{\omega \in \Omega_{j}^{*}(x)} G_{\omega} .
$$

Proof. The map $\Phi_{x}: \mathcal{U}_{N_{j}}(x) \rightarrow \mathbf{U}_{N_{j}}(x)$ is strictly increasing, hence bijective. Lemmas 2.12 .4 imply that

$$
\mathcal{U}_{N_{j}}(x) \subseteq I_{x_{1} \cdots x_{M+N_{j}} 0} \backslash \bigcup_{\omega \in \Omega_{j}^{*}(x)} G_{\omega} .
$$

For the converse inclusion, first we remove from the closed interval $I_{x_{1} \cdots x_{M+N_{j}} 0}$ the nonempty open interval $G_{x_{1} \cdots x_{M+N_{j}} 0}$ to obtain the union of two non-degenerate disjoint closed intervals $I_{x_{1} \cdots x_{M+N_{j}} 00}$ and $I_{x_{1} \cdots x_{M+N_{j}} 01}$. We emphasize that the non-empty of $G_{x_{1} \ldots x_{M+N_{j}} 0}$ follows by Lemma 2.4, since $N_{j} \geq 3$ and the word $x_{1} \ldots x_{M+N_{j}} 0$ ends with 10 by Lemma2.1, Then we proceed by induction. Assume that after a finite number of steps we get a disjoint union of non-degenerate closed intervals $I_{\omega}$, where $\omega$ runs over all length $n\left(>M+N_{j}\right)$ words of $\Omega_{j}(x)$. We will construct all level $n+1$ sub-intervals in the following way. If 
$I_{\omega 0} \quad I_{\omega 1}$

$G_{\omega}$

Figure 1: The geometrical structure of the basic intervals $I_{\omega}, I_{\omega 0}, I_{\omega 1}$ and the gap interval $G_{\omega}$.

$\omega \in \Omega_{j}^{*}(x)$, then we remove the open interval $G_{\omega}$, and replace $I_{\omega}$ by the two disjoint closed subintervals $I_{\omega 0}$ and $I_{\omega 1}$ (see Figure11). If $\omega \notin \Omega_{j}^{*}(x)$, then either $\omega 0 \in \Omega_{j}(x)$ or $\omega 1 \in \Omega_{j}(x)$. In this case we keep the interval $I_{\omega}$ with either $I_{\omega}=I_{\omega 0}$ or $I_{\omega}=I_{\omega 1}$.

Repeating this procedure indefinitely we construct the set $\mathcal{U}_{N_{j}}(x)$, and we obtain the converse inclusion

$$
I_{x_{1} \cdots x_{M+N_{j}} 0} \backslash \bigcup_{\omega \in \Omega_{j}^{*}(x)} G_{\omega} \subseteq \mathcal{U}_{N_{j}}(x) .
$$

Furthermore, we obtain that the gap intervals $G_{\omega}$ with $\omega \in \Omega_{j}^{*}(x)$ are pairwise disjoint.

\section{Proof of Theorem 1.1}

By Lemma 2.5 the Cantor set $\mathcal{U}_{N_{j}}(x)$ can be obtained by successively removing from the closed interval $I_{x_{1} \cdots x_{M+N_{j}} 0}$ a sequence of open intervals. By using the notation from Lemma 2.5 we define the thickness of $\mathcal{U}_{N_{j}}(x)$.

Definition 3.1. The thickness of $\mathcal{U}_{N_{j}}(x)$ is defined by

$$
\tau\left(\mathcal{U}_{N_{j}}(x)\right):=\inf _{\omega \in \Omega_{j}^{*}(x)}\left\{\frac{\left|I_{\omega 0}\right|}{\left|G_{\omega}\right|}, \frac{\left|I_{\omega 1}\right|}{\left|G_{\omega}\right|}\right\}
$$

where $|I|:=q-p$ denotes the length of a interval $I=[p, q]$.

We point out that the thickness given in Definition 3.1 coincides with that defined by Astels [1], and it is essentially the same as that defined by Newhouse [16]. Notice that the thickness is stable under non-trivial scaling, i.e., $\tau\left(\lambda \mathcal{U}_{N_{j}}(x)\right)=\tau\left(\mathcal{U}_{N_{j}}(x)\right)$ for all $\lambda \neq 0$. The following result follows from [1, Theorem 2.4].

Lemma 3.2. If $\tau\left(\mathcal{U}_{N_{j}}(x)\right) \geq 1$, then $\mathcal{U}_{N_{j}}(x)+\lambda \mathcal{U}_{N_{j}}(x)$ contains an interval for all $\lambda \neq 0$.

In view of the relation (2.2) and Lemma 3.2, the algebraic sum $\mathcal{U}(x)+\lambda \mathcal{U}(x)$ containing an interval will be proved if we find an index $j \geq 1$ such that $\tau\left(\mathcal{U}_{N_{j}}(x)\right) \geq 1$. For this we will compare the length of each non-degenerate interval $G_{\omega}$ with the lengths of its neighbors $I_{\omega 0}$ and $I_{\omega 1}$. We need three further lemmas; for the first one see also [10].

Henceforth we denote by $\varphi:=\frac{1+\sqrt{5}}{2}$ the Golden Ratio. 
Lemma 3.3. We have $\mathcal{U}(x) \subseteq(\varphi, 2]$ for all $x \in(0,1]$.

Proof. For $q \in(1, \varphi]$ only the endpoints of $[0,1 /(q-1)]$ have unique expansions, and they are outside $(0,1]$.

Next we establish some elementary inequalities.

Lemma 3.4. If the integers $m$ and $n$ are sufficiently large, then

$$
\left(1+\frac{1}{\varphi^{m}}\right)^{2 m}<\frac{\left(110^{\infty}\right)_{2}}{\left(\left(10^{n-1}\right)^{\infty}\right)_{\varphi}} \text { and }\left(1+\frac{1}{\varphi^{m}}\right)^{2 m}<\frac{\left(\left(1^{n-1} 0\right)^{\infty}\right)_{2}}{\left(\left(10^{n-3} 10\right)^{\infty}\right)_{\varphi}}
$$

Proof. The lemma follows from the following relations:

$$
\begin{aligned}
& \lim _{m \rightarrow \infty}\left(1+\frac{1}{\varphi^{m}}\right)^{2 m}=1, \\
& \lim _{n \rightarrow \infty}\left(\left(10^{n-1}\right)^{\infty}\right)_{\varphi}=\frac{1}{\varphi}<\frac{3}{4}=\left(110^{\infty}\right)_{2}
\end{aligned}
$$

and

$$
\lim _{n \rightarrow \infty}\left(\left(10^{n-3} 10\right)^{\infty}\right)_{\varphi}=\frac{1}{\varphi}<1=\lim _{n \rightarrow \infty}\left(\left(1^{n-1} 0\right)^{\infty}\right)_{2} .
$$

Lemma 3.5. Let $j \geq 1$ be sufficiently large. Then

$$
\left|G_{\omega}\right| \leq\left|I_{\omega 0}\right| \quad \text { and } \quad\left|G_{\omega}\right| \leq\left|I_{\omega 1}\right|
$$

for all $\omega \in \Omega_{j}^{*}(x)$.

Proof. Fix $\omega \in \Omega_{j}^{*}(x)$ of length $n\left(>M+N_{j}\right)$. Writing

$$
I_{\omega 0}=\left[q_{1}, q_{2}\right] \quad \text { and } \quad I_{\omega 1}=\left[q_{3}, q_{4}\right]
$$

we have to prove the inequalities

$$
q_{3}-q_{2} \leq q_{2}-q_{1} \quad \text { and } \quad q_{3}-q_{2} \leq q_{4}-q_{3}
$$

for some large integer $j$. By Lemma 2.3 it follows that

$$
\begin{array}{ll}
\omega\left(0^{N_{j}-1} 1\right)^{\infty} \preccurlyeq \Phi_{x}\left(q_{1}\right) \preccurlyeq \omega 0\left(10^{N_{j}-1}\right)^{\infty}, & \Phi_{x}\left(q_{2}\right)=\omega 0\left(1^{N_{j}-1} 0\right)^{\infty} ; \\
\omega 1\left(01^{N_{j}-1}\right)^{\infty} \preccurlyeq \Phi_{x}\left(q_{4}\right) \preccurlyeq \omega\left(1^{N_{j}-1} 0\right)^{\infty}, & \Phi_{x}\left(q_{3}\right)=\omega 1\left(0^{N_{j}-1} 1\right)^{\infty} .
\end{array}
$$

We emphasize by Lemma 2.5 that $q_{i} \in \mathcal{U}_{N_{j}}(x)$ for all $1 \leq i \leq 4$.

Bounds on $q_{2}-q_{1}$. First we give an upper bound of $q_{2}-q_{1}$. It follows from (3.1) that

$$
\left(\omega\left(01^{N_{j}-1}\right)^{\infty}\right)_{q_{2}}=x \geq\left(\omega\left(0^{N_{j}-1} 1\right)^{\infty}\right)_{q_{1}},
$$


whence

$$
\left(0^{n}\left(01^{N_{j}-1}\right)^{\infty}\right)_{q_{2}}-\left(0^{n}\left(0^{N_{j}-1} 1\right)^{\infty}\right)_{q_{1}} \geq\left(\omega 0^{\infty}\right)_{q_{1}}-\left(\omega 0^{\infty}\right)_{q_{2}} .
$$

Since $\omega=\omega_{1} \cdots \omega_{n}$ contains a non-zero digit $\omega_{\ell}=1$ for some $1 \leq \ell \leq M+1$ by Lemma 2.1 (ii), the right hand side may be minorized as follows:

$$
\left(\omega 0^{\infty}\right)_{q_{1}}-\left(\omega 0^{\infty}\right)_{q_{2}} \geq \frac{1}{q_{1}^{\ell}}-\frac{1}{q_{2}^{\ell}} \geq \frac{1}{q_{1} q_{2}^{\ell-1}}-\frac{1}{q_{2}^{\ell}}=\frac{q_{2}-q_{1}}{q_{1} q_{2}^{\ell}} \geq \frac{q_{2}-q_{1}}{q_{2}^{M+2}} .
$$

Combining the two estimates and using Lemma 2.1 (iii) we conclude that

$$
\begin{aligned}
q_{2}-q_{1} & \leq q_{2}^{M+2}\left(\left(0^{n}\left(01^{N_{j}-1}\right)^{\infty}\right)_{q_{2}}-\left(0^{n}\left(0^{N_{j}-1} 1\right)^{\infty}\right)_{q_{1}}\right) \\
& \leq q_{2}^{M+2}\left(0^{n}\left(01^{N_{j}-1}\right)^{\infty}\right)_{q_{2}} \leq \frac{q_{2}^{M+2}}{q_{2}^{n+1}}=\frac{1}{q_{2}^{n-M-1}} .
\end{aligned}
$$

Now we focus on the lower bound of $q_{2}-q_{1}$. We infer from (3.1) that

$$
\left(\omega 0\left(1^{N_{j}-1} 0\right)^{\infty}\right)_{q_{2}}=x \leq\left(\omega 0\left(10^{N_{j}-1}\right)^{\infty}\right)_{q_{1}},
$$

and this implies the estimate

$$
\begin{aligned}
\left(0^{n+1}\left(1^{N_{j}-1} 0\right)^{\infty}\right)_{q_{2}}-\left(0^{n+1}\left(10^{N_{j}-1}\right)^{\infty}\right)_{q_{1}} & \leq\left(\omega 0^{\infty}\right)_{q_{1}}-\left(\omega 0^{\infty}\right)_{q_{2}} \\
& \leq \sum_{i=1}^{\infty}\left(\frac{1}{q_{1}^{i}}-\frac{1}{q_{2}^{i}}\right)=\frac{q_{2}-q_{1}}{\left(q_{1}-1\right)\left(q_{2}-1\right)}
\end{aligned}
$$

Choosing by Lemma 3.4 a large integer $j_{0} \geq 1$ such that

$$
N_{j} \geq 4 \text { and }\left(1+\frac{1}{\varphi^{n-M}}\right)^{n+1}<\frac{\left(110^{\infty}\right)_{2}}{\left(\left(10^{N_{j}-1}\right)^{\infty}\right)_{\varphi}}
$$

for all $j \geq j_{0}$ and $n>M+N_{j}$, we deduce from the above estimate for all $j \geq j_{0}$ that

$$
\begin{aligned}
q_{2}-q_{1} & \geq(\varphi-1)^{2}\left(\left(0^{n+1}\left(1^{N_{j}-1} 0\right)^{\infty}\right)_{q_{2}}-\left(0^{n+1}\left(10^{N_{j}-1}\right)^{\infty}\right)_{q_{1}}\right) \\
& \geq(\varphi-1)^{2}\left(\left(0^{n+1}\left(1^{N_{j}-1} 0\right)^{\infty}\right)_{q_{2}}-\left(0^{n+1} 110^{\infty}\right)_{q_{2}}\right) \\
& \geq \frac{(\varphi-1)^{2}}{q_{2}^{n+4}} .
\end{aligned}
$$

Here the first inequality holds because $q_{2}>q_{1} \geq \varphi$ by Lemma 3.3 and the last inequality holds because $N_{j} \geq 4$. The crucial second inequality follows by (3.2), (3.3) and the 
inequality $q_{2}>q_{1} \geq \varphi$ :

$$
\begin{aligned}
\left(0^{n+1}\left(10^{N_{j}-1}\right)^{\infty}\right)_{q_{1}} & =\left(\frac{q_{2}}{q_{1}}\right)^{n+1} \frac{\left(\left(10^{N_{j}-1}\right)^{\infty}\right)_{q_{1}}}{q_{2}^{n+1}} \\
& \leq\left(1+\frac{q_{2}-q_{1}}{q_{1}}\right)^{n+1} \frac{\left(\left(10^{N_{j}-1}\right)^{\infty}\right)_{\varphi}}{q_{2}^{n+1}} \\
& \leq\left(1+\frac{1}{q_{1} q_{2}^{n-M-1}}\right)^{n+1} \frac{\left(\left(10^{N_{j}-1}\right)^{\infty}\right)_{\varphi}}{q_{2}^{n+1}} \\
& \leq\left(1+\frac{1}{\varphi^{n-M}}\right)^{n+1} \frac{\left(\left(10^{N_{j}-1}\right)^{\infty}\right)_{\varphi}}{q_{2}^{n+1}} \\
& <\frac{\left(110^{\infty}\right)_{2}}{q_{2}^{n+1}} \leq\left(0^{n+1} 110^{\infty}\right)_{q_{2}} .
\end{aligned}
$$

Bounds on $q_{4}-q_{3}$. We adapt the above arguments for $q_{2}-q_{1}$. First we give an upper bound of $q_{4}-q_{3}$. We infer from (3.1) that

$$
\left(\omega 1\left(0^{N_{j}-1} 1\right)^{\infty}\right)_{q_{3}}=x \leq\left(\omega\left(1^{N_{j}-1} 0\right)^{\infty}\right)_{q_{4}} .
$$

Since there exists $1 \leq \ell \leq M+1$ such that $\omega_{\ell}=1$ by Lemma 2.1 (ii), it follows that

$$
\begin{aligned}
\left(0^{n+1}\left(1^{N_{j}-2} 01\right)^{\infty}\right)_{q_{4}}-\left(0^{n+1}\left(0^{N_{j}-1} 1\right)^{\infty}\right)_{q_{3}} & \geq\left(\omega 10^{\infty}\right)_{q_{3}}-\left(\omega 10^{\infty}\right)_{q_{4}} \\
& \geq \frac{1}{q_{3}^{\ell}}-\frac{1}{q_{4}^{\ell}} \geq \frac{q_{4}-q_{3}}{q_{4}^{M+2}} .
\end{aligned}
$$

This implies that

$$
\begin{aligned}
q_{4}-q_{3} & \leq q_{4}^{M+2}\left(\left(0^{n+1}\left(1^{N_{j}-2} 01\right)^{\infty}\right)_{q_{4}}-\left(0^{n+1}\left(0^{N_{j}-1} 1\right)^{\infty}\right)_{q_{3}}\right) \\
& \leq q_{4}^{M+2}\left(0^{n+1}\left(1^{N_{j}-2} 01\right)^{\infty}\right)_{q_{4}} \leq \frac{q_{4}^{M+2}}{q_{4}^{n+1}}=\frac{1}{q_{4}^{n-M-1}},
\end{aligned}
$$

where the third inequality follows by Lemma 2.1 (iii) because $q_{4} \in \mathcal{U}_{N_{j}}(x)$.

Now we seek a lower bound of $q_{4}-q_{3}$. By Lemma 3.4 there exists $j_{1} \geq j_{0}$ (we use $j_{0}$ chosen in the first part of the proof) such that

$$
\left(1+\frac{1}{\varphi^{n-M}}\right)^{n+2}<\frac{\left(\left(1^{N_{j}-1} 0\right)^{\infty}\right)_{2}}{\left(\left(10^{N_{j}-3} 10\right)^{\infty}\right)_{\varphi}}
$$

for all $j \geq j_{1}$ and $n>M+N_{j}$. By (3.1) we have

$$
\left(\omega 1\left(0^{N_{j}-1} 1\right)^{\infty}\right)_{q_{3}}=x \geq\left(\omega 1\left(01^{N_{j}-1}\right)^{\infty}\right)_{q_{4}},
$$


whence

$$
\begin{aligned}
\left(0^{n+1}\left(01^{N_{j}-1}\right)^{\infty}\right)_{q_{4}}-\left(0^{n+1}\left(0^{N_{j}-1} 1\right)^{\infty}\right)_{q_{3}} & \leq\left(\omega 10^{\infty}\right)_{q_{3}}-\left(\omega 10^{\infty}\right)_{q_{4}} \\
& \leq \sum_{i=1}^{\infty}\left(\frac{1}{q_{3}^{i}}-\frac{1}{q_{4}^{i}}\right)=\frac{q_{4}-q_{3}}{\left(q_{4}-1\right)\left(q_{3}-1\right)} .
\end{aligned}
$$

Since $q_{4}>q_{3} \geq \varphi$ by Lemma 3.3, hence we deduce the following estimate of $q_{4}-q_{3}$ for all $j \geq j_{1}$ :

$$
\begin{aligned}
q_{4}-q_{3} & \geq(\varphi-1)^{2}\left(\left(0^{n+1}\left(01^{N_{j}-1}\right)^{\infty}\right)_{q_{4}}-\left(0^{n+1}\left(0^{N_{j}-1} 1\right)^{\infty}\right)_{q_{3}}\right) \\
& \geq(\varphi-1)^{2}\left(\left(0^{n+1}\left(010^{N_{j}-3} 1\right)^{\infty}\right)_{q_{3}}-\left(0^{n+1}\left(0^{N_{j}-1} 1\right)^{\infty}\right)_{q_{3}}\right) \\
& \geq \frac{(\varphi-1)^{2}}{q_{3}^{n+3}} .
\end{aligned}
$$

Here the crucial second inequality follows from (3.5) and (3.6):

$$
\begin{aligned}
\left(0^{n+1}\left(010^{N_{j}-3} 1\right)^{\infty}\right)_{q_{3}} & =\left(\frac{q_{4}}{q_{3}}\right)^{n+2} \frac{\left(\left(10^{N_{j}-3} 10\right)^{\infty}\right)_{q_{3}}}{q_{4}^{n+2}} \\
& \leq\left(1+\frac{q_{4}-q_{3}}{q_{3}}\right)^{n+2} \frac{\left(\left(10^{N_{j}-3} 10\right)^{\infty}\right)_{\varphi}}{q_{4}^{n+2}} \\
& \leq\left(1+\frac{1}{q_{3} q_{4}^{n-M-1}}\right)^{n+2} \frac{\left(\left(10^{N_{j}-3} 10\right)^{\infty}\right)_{\varphi}}{q_{4}^{n+2}} \\
& \leq\left(1+\frac{1}{\varphi^{n-M}}\right)^{n+2} \frac{\left(\left(10^{N_{j}-3} 10\right)^{\infty}\right)_{\varphi}}{q_{4}^{n+2}} \\
& <\frac{\left(\left(1^{N_{j}-1} 0\right)^{\infty}\right)_{2}}{q_{4}^{n+2}} \leq\left(0^{n+1}\left(01^{N_{j}-1}\right)^{\infty}\right)_{q_{4}} .
\end{aligned}
$$

Bounds on $q_{3}-q_{2}$. Note that

$$
\left(\omega 0\left(1^{N_{j}-1} 0\right)^{\infty}\right)_{q_{2}}=x=\left(\omega 1\left(0^{N_{j}-1} 1\right)^{\infty}\right)_{q_{3}}
$$

by (3.1). Since there exists $1 \leq \ell \leq M+1$ such that $\omega_{\ell}=1$ by Lemma 2.1 (ii), it follows that

$$
\left(0^{n} 1\left(0^{N_{j}-1} 1\right)^{\infty}\right)_{q_{3}}-\left(0^{n} 0\left(1^{N_{j}-1} 0\right)^{\infty}\right)_{q_{2}}=\left(\omega 0^{\infty}\right)_{q_{2}}-\left(\omega 0^{\infty}\right)_{q_{3}} \geq \frac{1}{q_{2}^{\ell}}-\frac{1}{q_{3}^{\ell}} \geq \frac{q_{3}-q_{2}}{q_{3}^{M+2}} .
$$

Using the inequalities $q_{2}<q_{3} \leq 2$ hence we infer that

$$
\begin{aligned}
q_{3}-q_{2} & \leq 2^{M+2}\left(\left(0^{n} 1\left(0^{N_{j}-1} 1\right)^{\infty}\right)_{q_{3}}-\left(0^{n} 0\left(1^{N_{j}-1} 0\right)^{\infty}\right)_{q_{2}}\right) \\
& \leq 2^{M+2}\left(\left(0^{n} 1\left(0^{N_{j}-1} 1\right)^{\infty}\right)_{q_{3}}-\left(0^{n} 0\left(1^{N_{j}-1} 0\right)^{\infty}\right)_{q_{3}}\right) \\
& \leq 2^{M+2}\left(\left(0^{n} 01^{N_{j}-1} 40^{\infty}\right)_{q_{3}}-\left(0^{n} 01^{N_{j}-1} 0^{\infty}\right)_{q_{3}}\right) \\
& =\frac{2^{M+4}}{q_{3}^{n+N_{j}+1}} .
\end{aligned}
$$


Here the crucial third inequality follows by

$$
\left(0^{n} 1\left(0^{N_{j}-1} 1\right)^{\infty}\right)_{q_{3}}<\left(0^{n+1}\left(1^{N_{j}-1} 2\right)^{\infty}\right)_{q_{3}}
$$

and the estimate

$$
\left(\left(1^{N_{j}-1} 2\right)^{\infty}\right)_{q_{3}}=\frac{\left(1^{N_{j}-1} 20^{\infty}\right) q_{3}}{1-q_{3}^{-N_{j}}} \leq \frac{1+q_{3}^{-N_{j}}}{1-q_{3}^{-N_{j}}} \leq \frac{1+\varphi^{-N_{j}}}{1-\varphi^{-N_{j}}} \leq 2,
$$

using that $\left(1^{N_{j}} 0^{\infty}\right)_{q_{3}} \leq 1, q_{3} \geq \varphi$ and $N_{j} \geq 3$.

Since $1<q_{2}<q_{3}$, we may choose $j_{2} \geq j_{1}$ such that

$$
2^{M+4} \leq(\varphi-1)^{2} q_{2}^{N_{j_{2}}-3} \leq(\varphi-1)^{2} q_{3}^{N_{j_{2}}-2}
$$

(The second inequality automatically follows from the first one.) Then, using also the relations (3.4) and (3.8), the following estimate holds for all $j \geq j_{2}$ :

$$
q_{3}-q_{2} \leq \frac{2^{M+4}}{q_{3}^{n+N_{j}+1}}<\frac{2^{M+4}}{q_{2}^{n+N_{j}+1}} \leq \frac{(\varphi-1)^{2}}{q_{2}^{n+4}} \leq q_{2}-q_{1} .
$$

Similarly, using (3.7) and (3.8) we obtain that

$$
q_{3}-q_{2} \leq \frac{2^{M+4}}{q_{3}^{n+N_{j}+1}}<\frac{(\varphi-1)^{2}}{q_{3}^{n+3}} \leq q_{4}-q_{3}
$$

for all $j \geq j_{2}$. Since the word $\omega$ was taken arbitrarily from $\Omega_{j}^{*}(x)$, this completes the proof.

Now we consider the algebraic product part of Theorem [1.1, By Lemma 3.3 we have $\mathcal{U}(x) \subset(\varphi, 2]$ for each $x \in(0,1]$. Then

$$
\mathcal{U}(x) \cdot \mathcal{U}(x)^{\lambda}=\left\{p q^{\lambda}: p, q \in \mathcal{U}(x)\right\}=\left\{e^{\ln p+\lambda \ln q}: p, q \in \mathcal{U}(x)\right\} .
$$

So, the algebraic product $\mathcal{U}(x) \cdot \mathcal{U}(x)^{\lambda}$ containing an interval is equivalent to that the algebraic sum $\ln \mathcal{U}(x)+\lambda \ln \mathcal{U}(x)$ contains an interval, where $\ln \mathcal{U}(x):=\{\ln q: q \in \mathcal{U}(x)\}$. Observe by Lemma 2.5 that for any $x \in(0,1]$ and any $j \geq 1$ the set $\mathcal{U}_{N_{j}}(x)$ is a Cantor subset of $\mathcal{U}(x)$. This implies that $\ln \mathcal{U}_{N_{j}}(x)$ is also a Cantor subset of $\ln \mathcal{U}(x)$. Combining this with Lemma 3.2 on the thickness we obtain the following

Lemma 3.6. For any given $x \in(0,1]$, if $\tau\left(\ln \mathcal{U}_{N_{j}}(x)\right) \geq 1$ for some $j \geq 1$, then $\mathcal{U}_{N_{j}}(x)$. $\mathcal{U}_{N_{j}}(x)^{\lambda}$ contains an interval for each non-zero real number $\lambda$.

Proof of Theorem [1.1. Fix $x \in(0,1]$ and $\lambda \neq 0$ arbitrarily. By Lemmas 3.2 and 3.5 it follows that the algebraic sum $\mathcal{U}(x)+\lambda \mathcal{U}(x)$ contains an interval. As for the algebraic product $\mathcal{U}(x) \cdot \mathcal{U}(x)^{\lambda}$ it suffices to show that $\tau\left(\ln \mathcal{U}_{N_{j}}(x)\right) \geq 1$ if $j$ is sufficiently large. Indeed, then the theorem will follow from Lemma 3.6 because of the inclusion (2.2). 
Fix $\omega \in \Omega_{j}^{*}(x)$ arbitrarily, of length $n\left(>M+N_{j}\right)$, and consider the intervals

$$
I_{\omega 0}=\left[q_{1}, q_{2}\right], \quad I_{\omega 1}=\left[q_{3}, q_{4}\right] \quad \text { and } \quad G_{\omega}=\left(q_{2}, q_{3}\right)
$$

as in the proof of Lemma 3.5. Then the corresponding basic intervals of level $n+1$ of $\ln \left(\mathcal{U}_{N_{j}}(x)\right)$ are

$$
\ln \left(I_{\omega 0}\right):=\left[\ln q_{1}, \ln q_{2}\right], \quad \ln \left(I_{\omega 1}\right):=\left[\ln q_{3}, \ln q_{4}\right] \quad \text { and } \quad \ln \left(G_{\omega}\right):=\left(\ln q_{2}, \ln q_{3}\right) .
$$

We have to prove that if $j$ is sufficiently large, then

$$
\ln q_{3}-\ln q_{2} \leq \ln q_{2}-\ln q_{1} \quad \text { and } \quad \ln q_{3}-\ln q_{2} \leq \ln q_{4}-\ln q_{3},
$$

or equivalently

$$
\frac{q_{3}}{q_{2}} \leq \frac{q_{2}}{q_{1}} \quad \text { and } \quad \frac{q_{3}}{q_{2}} \leq \frac{q_{4}}{q_{3}} .
$$

We use the estimates obtained in the proof of Lemma 3.5. If $j \geq j_{2}$, then we infer from (3.4) and (3.8) the relations

$$
\begin{aligned}
& \frac{q_{2}}{q_{1}} \geq 1+\frac{(\varphi-1)^{2}}{q_{1} q_{2}^{n+4}} \geq 1+\frac{(\varphi-1)^{2}}{q_{2}^{n+5}} \\
& \frac{q_{3}}{q_{2}} \leq 1+\frac{2^{M+4}}{q_{2} q_{3}^{n+N_{j}+1}} \leq 1+\frac{2^{M+4}}{q_{2}^{n+N_{j}+2}}
\end{aligned}
$$

Hence there exists $j_{3} \geq j_{2}$ such that

$$
\frac{q_{3}}{q_{2}} \leq 1+\frac{2^{M+4}}{q_{2}^{n+N_{j}+2}}<1+\frac{(\varphi-1)^{2}}{q_{2}^{n+5}} \leq \frac{q_{2}}{q_{1}}
$$

for all $j \geq j_{3}$, establishing the first inequality in (3.9).

Similarly, we deduce from (3.7) and (3.8) that

$$
\frac{q_{4}}{q_{3}} \geq 1+\frac{(\varphi-1)^{2}}{q_{3}^{n+4}} \quad \text { and } \quad \frac{q_{3}}{q_{2}} \leq 1+\frac{2^{M+4}}{q_{2} q_{3}^{n+N_{j}+1}}
$$

for all $j \geq j_{2}$. Hence, there exists $j_{4} \geq j_{3}$ such that

$$
\frac{q_{3}}{q_{2}} \leq 1+\frac{2^{M+4}}{q_{2} q_{3}^{n+N_{j}+1}}<1+\frac{(\varphi-1)^{2}}{q_{3}^{n+4}} \leq \frac{q_{4}}{q_{3}}
$$

for all $j \geq j_{4}$. This proves the second inequality in (3.9). 


\section{Proof of Theorem 1.3}

In this section we apply the symbolic Cantor sets constructed in Section 2 to the set $\mathcal{N}$ of non-matching parameters, and we prove Theorem 1.3. In order to describe the non-matching set $\mathcal{N}$ we recall the doubling map $D$ on the unit circle $[0,1)$ defined by

$$
D:[0,1) \rightarrow[0,1) ; \quad x \mapsto 2 x \quad(\bmod 1)
$$

The following characterization of $\mathcal{N}$ was implicitly given by [2].

Lemma 4.1. The following statements are equivalent:

(i) $\alpha \in \mathcal{N}$.

(ii) For all $n \geq 0$ we have

$$
D^{n}\left(\frac{1}{\alpha}\right) \notin\left(\frac{1}{2 \alpha}, 1-\frac{1}{2 \alpha}\right)
$$

(iii) $1 / \alpha \in[1 / 2,1]$ has a unique dyadic expansion $\left(a_{i}\right) \in\{0,1\}^{\mathbb{N}}$ satisfying

$$
\begin{cases}a_{n+1} a_{n+2} \cdots \preccurlyeq a_{1} a_{2} \cdots & \text { if } a_{n}=0 \\ a_{n+1} a_{n+2} \cdots \succcurlyeq\left(1-a_{1}\right)\left(1-a_{2}\right) \cdots & \text { if } a_{n}=1\end{cases}
$$

for all $n \geq 1$.

Proof. The equivalence of (i) and (ii) follows from [2]. As for (iii) $\Rightarrow$ (ii), let $\left(a_{i}\right)$ be the unique dyadic expansion of $1 / \alpha$. Then $\left(1-a_{i}\right)$ is the unique dyadic expansion of $1-1 / \alpha$. Hence, (ii) follows from (4.1).

To prove (ii) $\Rightarrow$ (iii), we first observe that the greedy dyadic expansion $\left(a_{i}\right)$ of $1 / \alpha$ cannot end with $10^{\infty}$, for otherwise there must exist $n \geq 0$ such that

$$
D^{n}\left(\frac{1}{\alpha}\right)=\frac{1}{2} \in\left(\frac{1}{2 \alpha}, 1-\frac{1}{2 \alpha}\right) .
$$

Hence, $1 / \alpha$ has a unique dyadic expansion $\left(a_{i}\right)$. Furthermore, (4.1) follows from the following observation: for each $n \geq 1$,

$$
D^{n-1}\left(\frac{1}{\alpha}\right) \leq \frac{1}{2 \alpha} \quad \Longleftrightarrow \quad a_{n}=0 \text { and } a_{n+1} a_{n+2} \ldots \preccurlyeq a_{1} a_{2} \ldots
$$

and

$$
D^{n-1}\left(\frac{1}{\alpha}\right) \geq 1-\frac{1}{2 \alpha} \quad \Longleftrightarrow \quad a_{n}=1 \text { and } a_{n+1} a_{n+2} \ldots \succcurlyeq\left(1-a_{1}\right)\left(1-a_{2}\right) \ldots
$$


Let $\mathbf{N}$ be the set of all sequences $\left(a_{i}\right) \in\{0,1\}^{\mathbb{N}}$ such that it is the unique dyadic expansion of $\left(\left(a_{i}\right)\right)_{2} \in[1 / 2,1]$ and it satisfies the inequalities in (4.1). Then by Lemma 4.1] it follows that the projection map

$$
\Psi: \mathbf{N} \rightarrow \mathcal{N} ; \quad\left(a_{i}\right) \mapsto \frac{1}{\left(\left(a_{i}\right)\right)_{2}}
$$

is well-defined. Indeed, $\Psi$ is bijective and strictly decreasing. Motivated by the symbolic Cantor sets constructed in Section 2, we will construct the symbolic Cantor subsets $\mathbf{N}_{m}$ contained in $\mathbf{N}$, such that the thickness of $\Psi\left(\mathbf{N}_{m}\right)$ is larger than 1 .

Given an integer $m \geq 3$, let $\mathbf{N}_{m}$ be the set of sequences $\left(a_{i}\right) \in\{0,1\}^{\mathbb{N}}$ satisfying

$$
a_{1} \cdots a_{m}=1^{m} \quad \text { and } \quad a_{n+1} \cdots a_{n+m} \notin\left\{0^{m}, 1^{m}\right\}
$$

for all $n \geq m$. Then each sequence $\left(a_{i}\right) \in \mathbf{N}_{m}$ satisfies (4.1) and ends with neither $01^{\infty}$ nor $10^{\infty}$. Hence, by Lemma 4.1 it follows that

$$
\mathbf{N}_{m} \subseteq \mathbf{N} \text { for all } m \geq 3
$$

By an analogous argument as in Lemmas 2.3 2.5, the set $\mathbf{N}_{m}$ is indeed a symbolic Cantor set and has a similar structure as $\mathbf{U}_{N_{j}}(x)$ as described in Section 2. Write $\mathcal{N}_{m}:=\Psi\left(\mathbf{N}_{m}\right)$. By Lemma 4.1 it follows that $\mathcal{N}_{m} \subset \mathcal{N}$ for all $m \geq 3$. Therefore it suffices to prove the thickness $\tau\left(\mathcal{N}_{m}\right) \geq 1$ for some large integer $m$.

In contrast with the definitions of the set $\Omega_{j}(x)$ of finite words and the symbolic intervals $\mathbf{I}_{\omega}$ in Section 2, we introduce the following notation. For $m \geq 3$, let $\Omega\left(\mathbf{N}_{m}\right)$ be the set of all finite initial words of length larger than $m$ occurring in $\mathbf{N}_{m}$. Given a word $\omega \in \Omega\left(\mathbf{N}_{m}\right)$, let $\mathbf{J}_{\omega}$ be the smallest symbolic interval containing all sequences of $\mathbf{N}_{m}$ that begin with $\omega$. Similarly to Lemma 2.3 , one can verify that the interval $\mathbf{J}_{\omega}$ has the form $\mathbf{J}_{\omega}=\left[\left(a_{i}\right),\left(b_{i}\right)\right]$ with $\left(a_{i}\right),\left(b_{i}\right) \in \mathbf{N}_{m}$. Notice that the map $\Psi$ is strictly decreasing on $\mathbf{N}_{m}$. Then we denote by $J_{\omega}=[p, q]$ the corresponding interval in $\mathbb{R}$, where $p=\Psi\left(\left(b_{i}\right)\right)$ and $q=\Psi\left(\left(a_{i}\right)\right)$.

Proof of Theorem 1.3. Fix a word $\omega \in \Omega\left(\mathbf{N}_{m}\right)$ of length $n(>m)$ such that the open interval $O_{\omega}:=J_{\omega} \backslash\left(J_{\omega 0} \cup J_{\omega 1}\right) \neq \emptyset$. Write

$$
J_{\omega}=J_{\omega 1} \cup O_{\omega} \cup J_{\omega 0}=:\left[p_{1}, p_{2}\right] \cup\left(p_{2}, p_{3}\right) \cup\left[p_{3}, p_{4}\right]
$$

Notice that the map $\Psi$ is strictly decreasing. By Lemma 2.3 it follows that

$$
\begin{array}{ll}
\Psi\left(\omega\left(1^{m-1} 0\right)^{\infty}\right) \leq p_{1} \leq \Psi\left(\omega 1\left(01^{m-1}\right)^{\infty}\right), & p_{2}=\Psi\left(\omega 1\left(0^{m-1} 1\right)^{\infty}\right) \\
\Psi\left(\omega 0\left(10^{m-1}\right)^{\infty}\right) \leq p_{4} \leq \Psi\left(\omega\left(0^{m-1} 1\right)^{\infty}\right), & p_{3}=\Psi\left(\omega 0\left(1^{m-1} 0\right)^{\infty}\right) .
\end{array}
$$

By the thickness as described in Lemmas $[3.2$, in order to prove Theorem 1.3 (i) it suffices to prove the inequalities

$$
p_{3}-p_{2} \leq p_{2}-p_{1} \quad \text { and } \quad p_{3}-p_{2} \leq p_{4}-p_{3}
$$


for some large integer $m$.

By (4.2) it follows that

$$
\begin{aligned}
p_{2}-p_{1} & \geq \Psi\left(\omega 1\left(0^{m-1} 1\right)^{\infty}\right)-\Psi\left(\omega 1\left(01^{m-1}\right)^{\infty}\right) \\
& =\frac{1}{\left(\omega 1\left(0^{m-1} 1\right)^{\infty}\right)_{2}}-\frac{1}{\left(\omega 1\left(01^{m-1}\right)^{\infty}\right)_{2}} \geq \frac{\left(0^{n+2} 10^{\infty}\right)_{2}}{\left(\left(\omega 110^{\infty}\right)_{2}\right)^{2}}, \\
p_{4}-p_{3} & \geq \Psi\left(\omega 0\left(10^{m-1}\right)^{\infty}\right)-\Psi\left(\omega 0\left(1^{m-1} 0\right)^{\infty}\right) \\
& =\frac{1}{\left(\omega 0\left(10^{m-1}\right)^{\infty}\right)_{2}}-\frac{1}{\left(\omega 0\left(1^{m-1} 0\right)^{\infty}\right)_{2}} \geq \frac{\left(0^{n+2} 10^{\infty}\right)_{2}}{\left(\left(\omega 110^{\infty}\right)_{2}\right)^{2}}
\end{aligned}
$$

and

$$
\begin{aligned}
p_{3}-p_{2} & =\Psi\left(\omega 0\left(1^{m-1} 0\right)^{\infty}\right)-\Psi\left(\omega 1\left(0^{m-1} 1\right)^{\infty}\right) \\
& =\frac{1}{\left(\omega 0\left(1^{m-1} 0\right)^{\infty}\right)_{2}}-\frac{1}{\left(\omega 1\left(0^{m-1} 1\right)^{\infty}\right)_{2}} \leq \frac{\left(0^{n+m} 30^{\infty}\right)_{2}}{\left(\left(\omega 010^{\infty}\right)_{2}\right)^{2}} .
\end{aligned}
$$

Take $m_{0} \geq 3$ such that

$$
\frac{\left(0^{n+m} 30^{\infty}\right)_{2}}{\left(0^{n+2} 10^{\infty}\right)_{2}}<\frac{1}{2}\left(\frac{\left(\omega 010^{\infty}\right)_{2}}{\left(\omega 110^{\infty}\right)_{2}}\right)^{2}
$$

for all $m \geq m_{0}$. Here the existence of $m_{0}$ follows from that the left term of (4.4) tends to zero as $m \rightarrow \infty$, while the right term is a positive constant independent of $m$. Then (4.4) and the estimates of $p_{2}-p_{1}, p_{4}-p_{3}, p_{3}-p_{2}$ imply (4.3) for all $m \geq m_{0}$ :

$$
p_{3}-p_{2} \leq \frac{\left(0^{n+m} 30^{\infty}\right)_{2}}{\left(\left(\omega 010^{\infty}\right)_{2}\right)^{2}}<\frac{\left(0^{n+2} 10^{\infty}\right)_{2}}{\left(\left(\omega 110^{\infty}\right)_{2}\right)^{2}} \leq \min \left\{p_{2}-p_{1}, p_{4}-p_{3}\right\} \text {. }
$$

Applying Lemma 3.2 we conclude that $\mathcal{N}_{m}+\lambda \mathcal{N}_{m}$ contains an interval for all $\lambda \neq 0$ and any $m \geq m_{0}$.

Next, since $1 \leq p_{1}<p_{2}<p_{3} \leq 2$, we also infer from (4.4) and the estimates of $p_{2}-p_{1}, p_{4}-p_{3}, p_{3}-p_{2}$ for all $m \geq m_{0}$ the relations

$$
\frac{p_{3}}{p_{2}} \leq 1+\frac{\left(0^{n+m} 30^{\infty}\right)_{2}}{p_{2}\left(\left(\omega 010^{\infty}\right)_{2}\right)^{2}}<1+\frac{\left(0^{n+2} 10^{\infty}\right)_{2}}{p_{1}\left(\left(\omega 110^{\infty}\right)_{2}\right)^{2}} \leq \frac{p_{2}}{p_{1}}
$$

and

$$
\frac{p_{3}}{p_{2}} \leq 1+\frac{\left(0^{n+m} 30^{\infty}\right)_{2}}{p_{2}\left(\left(\omega 010^{\infty}\right)_{2}\right)^{2}}<1+\frac{\left(0^{n+2} 10^{\infty}\right)_{2}}{p_{3}\left(\left(\omega 110^{\infty}\right)_{2}\right)^{2}} \leq \frac{p_{4}}{p_{3}} .
$$

Applying Lemma 3.6 we conclude that the algebraic product $\mathcal{N}_{m} \cdot \mathcal{N}_{m}^{\lambda}$ contains an interval for all $\lambda \neq 0$ and any $m \geq m_{0}$.

Since $\mathcal{N}_{m} \subset \mathcal{N}$ for all $m \geq 3$, this completes the proof. 


\section{Final remarks}

The method used in the proofs of Theorems 1.1 and 1.3 can also be applied to many other Cantor sets that come up in dynamics. In this section we continue the investigation of the algebraic sum and product of $\mathcal{U}(x)$ for $x=1$. Recall that $\mathcal{U}(1)$ is the set of univoque bases $q \in(1,2]$ such that 1 has a unique $q$-expansion. As it is customory, let us simply write $\mathcal{U}$ instead of $\mathcal{U}(1)$.

Since both $\mathcal{U}+\mathcal{U}$ and $\mathcal{U} \cdot \mathcal{U}$ contain an interval by Theorem 1.1, it is natural to ask whether $\mathcal{U}+\mathcal{U}$ and $\mathcal{U} \cdot \mathcal{U}$ themselves are intervals. The answer is negative:

Proposition 5.1. Neither $\mathcal{U}+\mathcal{U}$, nor $\mathcal{U} \cdot \mathcal{U}$ is an interval. The same conclusion holds if we replace $\mathcal{U}$ by its topological closure $\overline{\mathcal{U}}$.

Before proving Proposition 5.1 we recall some results from [3, [5, 8, 9$]$ on the topological properties of $\mathcal{U}$. First, $\overline{\mathcal{U}}$ is a Cantor set and $q_{K L} \approx 1.78723$ is its smallest element. Next, we have

$$
\overline{\mathcal{U}}=\left[q_{K L}, 2\right] \backslash \bigcup\left(q_{L}, q_{R}\right)
$$

where on the right-hand side we have a union of countably many pairwise disjoint open intervals: the connected components of $\left[q_{K L}, 2\right] \backslash \overline{\mathcal{U}}$.

Furthermore, for each of these intervals $\left(q_{L}, q_{R}\right)$ there exists a word $a_{1} \cdots a_{m}$ with $a_{m}=0$, satisfying the lexicographic inequalities

$$
\left(\overline{a_{1} \cdots a_{m}}\right)^{\infty} \prec \sigma^{i}\left(\left(a_{1} \cdots a_{m}\right)^{\infty}\right) \preccurlyeq\left(a_{1} \cdots a_{m}\right)^{\infty} \quad \text { for all } \quad i \geq 0
$$

and the equalities

$$
\Phi_{1}\left(q_{L}\right)=\left(a_{1} \cdots a_{m}\right)^{\infty} \quad \text { and } \quad \Phi_{1}\left(q_{R}\right)=a_{1} \cdots a_{m}^{+} \overline{a_{1} \cdots a_{m}} \overline{a_{1} \cdots a_{m}^{+}} a_{1} \cdots a_{m}^{+} \cdots .
$$

Here $\sigma$ denotes the usual left-shift operator, and we use the notations

$$
\overline{a_{1} \cdots a_{m}}:=\left(1-a_{1}\right) \cdots\left(1-a_{m}\right), \quad a_{1} \cdots a_{m}^{+}:=a_{1} \cdots a_{m-1}\left(a_{m}+1\right) .
$$

We recall that the left endpoints $q_{L}$ are algebraic integers, while the right endpoints $q_{R}$, called de Vries-Komornik numbers in [11], are transcendental and their expansions $\Phi_{1}\left(q_{R}\right)$ are Thue-Morse type sequences.

We also need an elementary lemma:

Lemma 5.2. Let $A$ be a non-empty set of real numbers, and set

$$
a:=\inf A, \quad b:=\sup A .
$$

If there exists a non-empty subinterval $(c, d)$ of $(a, b)$ such that

$$
A \cap(c, d)=\emptyset \text { and } \quad d-c>c-a,
$$

then $A+A$ is not an interval. 
Proof. Since $A+A$ meets both a neighborhood of both $2 a$ and $2 b$ by the definition of the infimum and supremum, it suffices to show that it does not meet the non-empty subinterval $(2 c, a+d)$.

Let $x, y \in A$. If $x \leq c$ and $y \leq c$, then $x+y \leq 2 c$. Otherwise at least one of them is at least $d$. Since the other one is at least $a$, then $x+y \geq a+d$.

Proof of Proposition 5.1. In order to prove that $\mathcal{U}+\mathcal{U}$ is not an interval, by the preceding lemma it suffices to find a connected component $\left(q_{L}, q_{R}\right)$ of $\left[q_{K L}, 2\right] \backslash \overline{\mathcal{U}}$ satisfying

$$
q_{R}-q_{L}>q_{L}-q_{K L}
$$

We claim that the interval $\left(q_{L}, q_{R}\right)$ associated with the word $a_{1} \cdots a_{6}=110100$ satisfies this inequality.

This word defines an interval $\left(q_{L}, q_{R}\right)$ indeed, because it satisfies the inequalities in (5.1):

$$
(001011)^{\infty} \prec \sigma^{i}\left((110100)^{\infty}\right) \preccurlyeq(110100)^{\infty} \text { for all } i \geq 0 .
$$

In view of (5.2) the endpoints of $\left(q_{L}, q_{R}\right)$ satisfy the relations

$$
\Phi_{1}\left(q_{L}\right)=(110100)^{\infty} \text { and } \Phi_{1}\left(q_{R}\right)=110101001011001010110101 \cdots .
$$

By a numerical calculation we have $q_{L} \approx 1.78854$ and $q_{R} \approx 1.79656$. Hence

$$
q_{R}-q_{L}>1.79654-1.78854=0.008
$$

and

$$
q_{L}-q_{K L} \approx 1.78854-1.78723=0.00131,
$$

so that the inequality (5.3) is satisfied. The above proof remains valid for $\overline{\mathcal{U}}+\overline{\mathcal{U}}$ instead of $\mathcal{U}+\mathcal{U}$

Next we consider the product $\mathcal{U} \cdot \mathcal{U}$. Since it is homeomorphic to

$$
\ln \mathcal{U}+\ln \mathcal{U}=\{\ln p+\ln q: p, q \in \mathcal{U}\},
$$

it suffices to find a connected component $\left(q_{L}, q_{R}\right)$ of $\left[q_{K L}, 2\right] \backslash \overline{\mathcal{U}}$ satisfying

$$
\ln q_{R}-\ln q_{L}>\ln q_{L}-\ln q_{K L}, \quad \text { i.e., } \quad \frac{q_{R}}{q_{L}}>\frac{q_{L}}{q_{K L}} .
$$

This is satisfied with the same interval $\left(q_{L}, q_{R}\right) \approx(1.78854,1.79656)$ as in the first part of the proof because

$$
\frac{q_{R}}{q_{L}} \approx 1.00448>1.00073 \approx \frac{q_{L}}{q_{K L}}
$$

by a numerical computation. The proof remains valid for $\overline{\mathcal{U}} \cdot \overline{\mathcal{U}}$ instead of $\mathcal{U} \cdot \mathcal{U}$.

We end our paper with the following

Conjecture 5.3. Both the algebraic difference $\mathcal{U}-\mathcal{U}$ and quotient $\mathcal{U} \cdot \mathcal{U}^{-1}$ are intervals. The same conclusion holds if we replace $\mathcal{U}$ by its topological closure $\overline{\mathcal{U}}$. 


\section{Acknowledgements}

The authors thank the anonymous referee for improving the presentation of the paper. The second author was sponsored by NWO visitor's travel grant 040.11.599. The third author was supported by NSFC No. 11401516. The fourth author was supported by NSFC No. 11671147, 11571144 and Science and Technology Commission of Shanghai Municipality (STCSM) No. 13dz2260400.

\section{References}

\section{References}

[1] S. Astels, Cantor sets and numbers with restricted partial quotients, Trans. Amer. Math. Soc. 352 (1) (2000) 133-170.

[2] K. Dajani, C. Kalle, Invariant measures, matching and the frequency of 0 for signed binary expansions, arXiv:1703.06335 (2017).

[3] M. de Vries, V. Komornik, Unique expansions of real numbers, Adv. Math. 221 (2009) $390-427$.

[4] M. de Vries, V. Komornik, Expansions in non-integer bases, In Combinatorics, words and symbolic dynamics, volume 159 of Encyclopedia Math. Appl., pages 18-58. Cambridge Univ. Press, Cambridge, 2016.

[5] M. de Vries, V. Komornik, P. Loreti, Topology of the set of univoque bases, Topology Appl. 205 (2016) 117-137.

[6] P. Erdős, I. Joó, On the number of expansions $1=\sum q^{-n_{i}}$, Ann. Univ. Sci. Budapest. Eötvös Sect. Math. 35 (1992) 129-132.

[7] V. Komornik, Expansions in noninteger bases, Integers 11B (2011) Paper No. A9, $1-30$.

[8] V. Komornik, P. Loreti, Unique developments in non-integer bases, Amer. Math. Monthly 105 (7) (1998) 636-639.

[9] V. Komornik, P. Loreti, On the topological structure of univoque sets, J. Number Theory 122 (1) (2007) 157-183.

[10] D. Kong, On small univoque bases of real numbers, Acta Math. Hungar. 150 (1) (2016) 194-208.

[11] D. Kong, W. Li, Hausdorff dimension of unique beta expansions, Nonlinearity 28 (1) (2015) 187-209.

[12] D. Kong, W. Li, F. Lü, M. de Vries, Univoque bases and Hausdorff dimension. Monatsh. Math. 184 (3) (2017) 443-458. 
[13] R. L. Kraft, Intersections of thick Cantor sets, Mem. Amer. Math. Soc. 97 (468) (1992) vi+119.

[14] R. L. Kraft, Random intersections of thick Cantor sets, Trans. Amer. Math. Soc. 352 (3) (2000) 1315-1328.

[15] F. Lü, B. Tan, J. Wu, Univoque sets for real numbers, Fund. Math. 227 (1) (2014) 69-83.

[16] S. E. Newhouse, The abundance of wild hyperbolic sets and nonsmooth stable sets for diffeomorphisms, Inst. Hautes Études Sci. Publ. Math. (50) (1979) 101-151.

[17] W. Parry, On the $\beta$-expansions of real numbers, Acta Math. Acad. Sci. Hungar. 11 (1960) 401-416.

[18] A. Rényi, Representations for real numbers and their ergodic properties, Acta Math. Acad. Sci. Hungar. 8 (1957) 477-493.

[19] N. Sidorov, Almost every number has a continuum of $\beta$-expansions, Amer. Math. Monthly 110 (9) (2003) 838-842. 